\title{
HIMENÓPTEROS ASSOCIADOS A SOLANUM GILO RADDI (SOLANACEAE)
}

\author{
Marcelo Picanço ${ }^{1}$ \\ Vicente Wagner Dias Casali ${ }^{2}$ \\ Ivênio Rubens de Oliveira ${ }^{1}$ \\ Germano Leão Demolin Leite ${ }^{1}$
}

\begin{abstract}
Hymenoptera associated to Solanum gilo (Solanaceae). The Hymenoptera fauna associated to Solanum gilo was studied. There were 21 species of Hymenoptera from eight families. It was observed Homalotylus flaminus (Dalman, 1820) (Encyrtidae) parasiting the adults of Cycloneda sanguinea Linnaeus, 1763 (Coleoptera, Coccinellidae) and Chalcididae parasiting caterpillars of Mechanitis polymnia casabranca Haensch, 1905 (Lepidoptera, Nymphalidae, Ithomiinae, Sthomiinae). Vespidae were observed preying both M. polymnia casabranca and Diabrotica speciosa Germar, 1824 (Coleoptera, Chrysomelidae) adults. Halictidae, Andrenidae and Anthophoridae, were observed visiting flowers and Trigona spinipes (Fabricius, 1793) (Apidae) attacking fruits and stem apices. The most abundant Hymenoptera were Formicidae; Crematogaster sp. and Camponotus rufipes (Fabricus, 1775) were observed in protocooperation relation with Homoptera, the main cause of plant mortality was Solenopsis saevissima (Smith, 1855). Higher population density of Formicidae occurred on the final phase of cultivation, when there were high insolation and temperature and low pluvial precipitations.

KEY WORDS. Insecta, entomofauna, population dynamics, protocooperation
\end{abstract}

Estudos sobre populações de insetos-praga e seus inimigos naturais, são de fundamental importância para o estabelecimento de base ecológica dos programas de manejo integrado de pragas, propiciando assim, a adoção de táticas de controle que sejam menos prejudiciais ao meio ambiente e à saúde humana (PEDIGO 1989; DENT 1993).

Apesar do consumo dos frutos do jiloeiro, Solanum gilo (Raddi, 1825), ser bastante difundido no Brasil, esta olerícola é pouco estudada (FILGUEIRA 1982), existindo poucas informações sobre suas pragas (MENEZES 1978). Entretanto é grande a utilização de agrotóxicos na cultura (na maioria das vezes de forma indiscriminada), devido aos riscos da atividade, alto investimento e exigências dos consumidores em termos de aparência dos frutos (VILAS-BôAS \& CASTELO BRANCO 1990).

1) Departamento de Biologia Animal, Universidade Federal de Viçosa, 36571-000, Viçosa, Minas Gerais, Brasil.

2) Departamento de Fitotecnia, Universidade Federal de Viçosa, 36571-000, Viçosa, Minas Gerais, Brasil. 
Neste contexto, torna-se importante a obtenção de informações sobre a entomofauna associada ao jiloeiro; assim, constituíram-se objetivos deste trabalho, a determinação das espécies de himenópteros associadas ao jiloeiro, descrição de seus hábitos alimentares e monitoramento da flutuação da densidade populacional das espécies mais abundantes, em Viçosa, Minas Gerais.

\section{MATERIAL E MÉTODOS}

A pesquisa foi realizada no Campus da Universidade Federal de Viçosa, no período de 01-XI-1984 a 31-X-1985. Foram realizados dois plantios de jiloeiro em 01-XI-1984 e 14-V-1985, ocupando cada um área de $130 \mathrm{~m}^{2}$. O semeio em canteiros foi realizado em sulcos distanciados de $15 \mathrm{~cm}$, usando-se $2 \mathrm{~g} / \mathrm{m}^{2}$ de sementes de jiloeiro da cultivar Tinguá. Quando as plântulas atingiram $20 \mathrm{~cm}$ de altura foram transplantadas para covas espaçadas de $1 \times 1 \mathrm{~m}$ no campo, previamente preparado por meio de aração e gradagem (FILGUEIRA 1982).

Durante a condução dos cultivos, a cada três dias, a himenopterofauna do dossel foi estudada em 10 plantas, casualizadas previamente, por meio de contagens das espécies, suas densidades, fases no desenvolvimento e hábito alimentar. Também monitorou-se a mortalidade das plantas (níveis e causas). A cada 15 dias, quatro plantas previamente casualizadas foram coletadas juntamente com um paralelepípedo de material de solo com dimensões de $8 \times 8 \times 15 \mathrm{~cm}$. Cada paralelepípedo foi colocado em funil Berlese durante 72 horas, para a coleta, contagem e identificação da himenopterofauna subterrânea. Os dados diários de temperatura média, umidade relativa do ar, insolação e total de precipitação pluvial foram obtidos na Estação Climatológica Principal (INEMET/5DISME/UFV). A identificação dos Encyrtidae e Formicidae foi realizada pelo Dr. Leland Chandler (in memorian); os Vespidae pela Dra. Maria Cristina de Almeida (Universidade Federal do Paraná, Curitiba, Paraná) e os Halictidae, Andrenidae, Anthophoridae e Apidae pelo Dr. José Ricardo Cure Hakin (Universidade Militar de Nova Granada, Bogotá, Colômbia).

Confeccionaram-se curvas de flutuação da densidade populacional das espécies de Hymenoptera mais abundantes. Realizaram-se análises de correlação de Pearson $(\mathrm{P}<0,05)$ entre as densidades populacionais das espécies de Hymenoptera mais abundantes com os elementos climáticos.

\section{RESULTADOS E DISCUSSÃO}

Observou-se Homalotylus flaminus (Dalman, 1820) (Encyrtidae) parasitando adultos do predador Cycloneda sanguinea Linnaeus, 1763 (Coleoptera, Coccinellidae). Após ter completado a fase larval, o parasitóide perfurou o exoesqueleto de $C$. sanguinea e na face ventral do corpo deste, confeccionou casulo onde empupou-se. Os fios de seda do casulo fixaram o corpo do predador à planta, impedindo-o de se movimentar e caçar suas presas, levando-o à morte por inanição. Este Encyrtidae atuou como consumidor terciário na cadeia alimentar, como foi observado por SANTOS \& PINTO (1981). Verificou-se Chalcididae parasitando lagartas de Mechanitis polymnia casabranca Haensch, 1905 (Lepidoptera, Nym- 
phalidae, Ithomiinae, Sthomiinae). Espécies de Vespidae [Polistes versicolor versicolor (Oliver, 1791), Polybia flavifrons hecuba (Richards, 1951), Polybia occidentalis (Oliver, 1791), Polybia scutellaris (White, 1841), Polybia tinctipennis tinctipennis (Fox, 1898), Protonectarina sylveirae (De Saussure, 1854)] foram observadas predando lagartas de $M$. polymnia casabranca e adultos de Diabrotica speciosa Germar, 1824 (Coleoptera, Chrysomelidae) (Tab. I).

Tabela I. Himenopterofauna associada ao jiloeiro. Viçosa, Minas Gerais, 1984 e 1985.

\begin{tabular}{|c|c|c|c|}
\hline Familia & Espécies & Fases observadas & Grupo de nicho ecológico \\
\hline Encyrtidae & Homalotylus flaminus (Dalman, 1820) & Larval, pupal e adulta & Parasitóides \\
\hline Chalcididae & Uma espécie não identificada & Adulta & Parasitóides \\
\hline \multirow[t]{9}{*}{ Formicidae } & Acromyrmex Mayr, 1865 & Adulta & Associada às raizes \\
\hline & Brachymyrmex sp. Mayr, 1868 & Adulta & Associada às raizes \\
\hline & Camponotus rufipes (Fabr., 1775) & Adulta & $\begin{array}{l}\text { Associada às raizes e em } \\
\text { procooperaçăo com Homoptera na } \\
\text { parte aérea }\end{array}$ \\
\hline & Crematogaster sp. Lund, 1831 & Adulta & $\begin{array}{l}\text { Procooperaçăo com Homoptera na } \\
\text { parte aérea }\end{array}$ \\
\hline & Hypoponera sp. Santschi, 1938 & Adulta & Associada às raizes \\
\hline & Mycocepurus smithi Forel, 1893 & Adulta & Associada às raizes \\
\hline & Pheidole sp. Westwood, 1841 & Adulta & Associada às raizes \\
\hline & Solenopsis saevissima (Smith, 1855) & Adulta & Causadora de mortalidade de plantas \\
\hline & Wasmannia auropunctata (Roger, 1863) & Adulta & Associada às raizes \\
\hline \multirow[t]{6}{*}{ Vespidae } & Polistes versicolor versicolor (Olivier, 1791) & Adulta & Predadora \\
\hline & Polybia flavifrons hecuba (Richards, 1951) & Adulta & Predadora \\
\hline & Polybia occidentalis (Oliver, 1791) & Adulta & Predadora \\
\hline & Polybia scutellaris (White, 1841) & Adulta & Predadora \\
\hline & Polybia tinctipennis tinctipennis (Fox, 1898) & Adulta & Predadora \\
\hline & Protonectarina sylveirae (De Saussure, 1854) & Adulta & Predadora \\
\hline Halictidae & Augochloropsis sp. Cockerell, 1897 & Adulta & Polinizadora \\
\hline Andrenidae & Oxaea flavescens Klug, 1807 & Adulta & Polinizadora \\
\hline \multirow[t]{3}{*}{ Anthophoridae } & Exomalopsis sp. Spinola, 1853 & Adulta & Polinizadora \\
\hline & Thygater analis (Lepeletier, 1841) & Adulta & Polinizadora \\
\hline & Duas espécies não identificadas & Adulta & Polinizadora \\
\hline Apidae & Trigona spinipes (Fabr., 1793) & Adulta & Ataque a frutos e ápices caulinares \\
\hline
\end{tabular}

Adultos de Augochloropsis sp. (Halictidae); Oxaea flavescens Klug., 1807 (Andrenidae); Exomalopsis sp. e Thygater analis (Lepeletier, 1841) (Anthophoridae), foram observados polinizando flores do jiloeiro (Tab. I). SILVEIRA \& CAMPOS (1995), observaram estas espécies visitando flores de Solanum spp. em ecossistema de cerrado. Como as plantas do gênero Solanum possuem anteras poricídas para coleta de pólen, estas abelhas vibravam as anteras pela transmissão de contrações de músculos indiretos de vôo, o que produzia som que podia ser ouvido a certa distância (MiCHENER 1962; BUCHMANN 1983). Observou-se adultos de Trigona spinipes (Fabricius, 1793) (Apidae) atacando os frutos e cascas dos ápices caulinares das plantas (Tab. I). Esta espécie é relatada como praga de culturas como: abiu, bananeira, citros, jaboticabeira, jaqueira, mangueira, pinheiro-do-paraná e roseira atacando flores, frutos, brotações e folhas novas para coleta de resinas empregadas na construção de seus ninhos GALLO et al. (1988).

Entre os Hymenoptera, as espécies mais abundantes foram da família Formicidae. Entre estas, Solenopsis saevissima (Smith, 1855) foi a principal causadora de mortalidade de plantas jovens de jiloeiro $(0,77 \%$ no primeiro cultivo e $75,30 \%$ no segundo cultivo) devido à destruição total ou parcial da casca e medula do caule, 
o que deixa tais tecidos expostos a ação de fitopatógenos. Os ninhos desta espécie se localizavam na base do caule das plantas. Tais fatos também foram relatados por GALlo et al. (1988) nas culturas de batatinha, moranguinho e quiabeiro.

Sua densidade populacional foi mais elevada no sistema radicular do que na parte aérea das plantas. Sua população na parte aérea foi mais elevada nos meses de maio e junho, período caracterizado pela ausência de chuvas, alta insolação e baixa temperatura. No sistema radicular as densidades mais elevadas, desta espécie, ocorreram em períodos de elevada insolação, baixas precipitações pluviais e umidade relativa do ar (Figs 1, 2, Tab. II). FowLER (1979) observou que o número e o comprimento de colônias de Labidus praedator Smith, 1858 (Formicidae) foram maiores em períodos de menor Umidade relativa do ar.
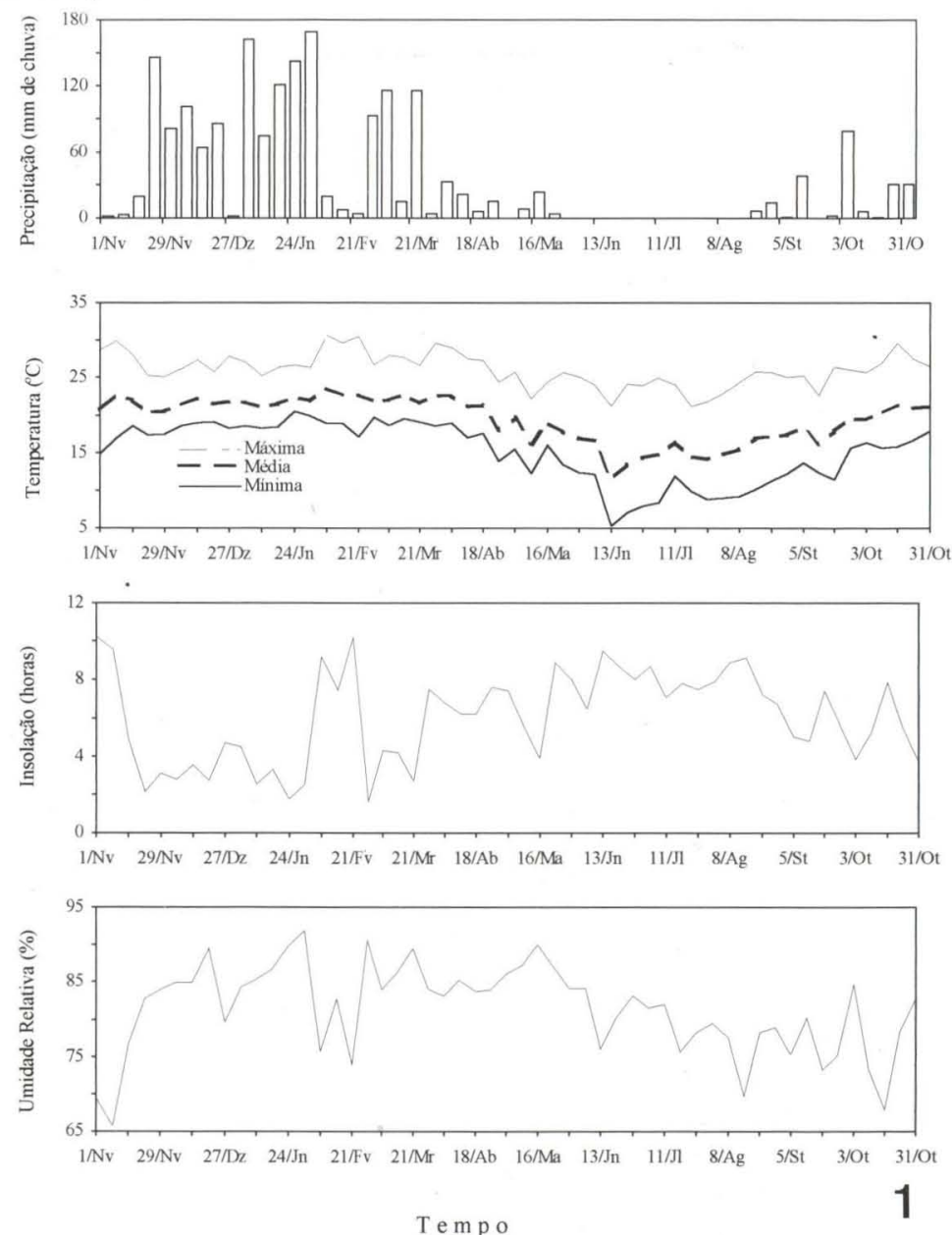

Fig. 1. Dados meteorológicos de Viçosa, Mınas Gerais, 1984 e 1985. 


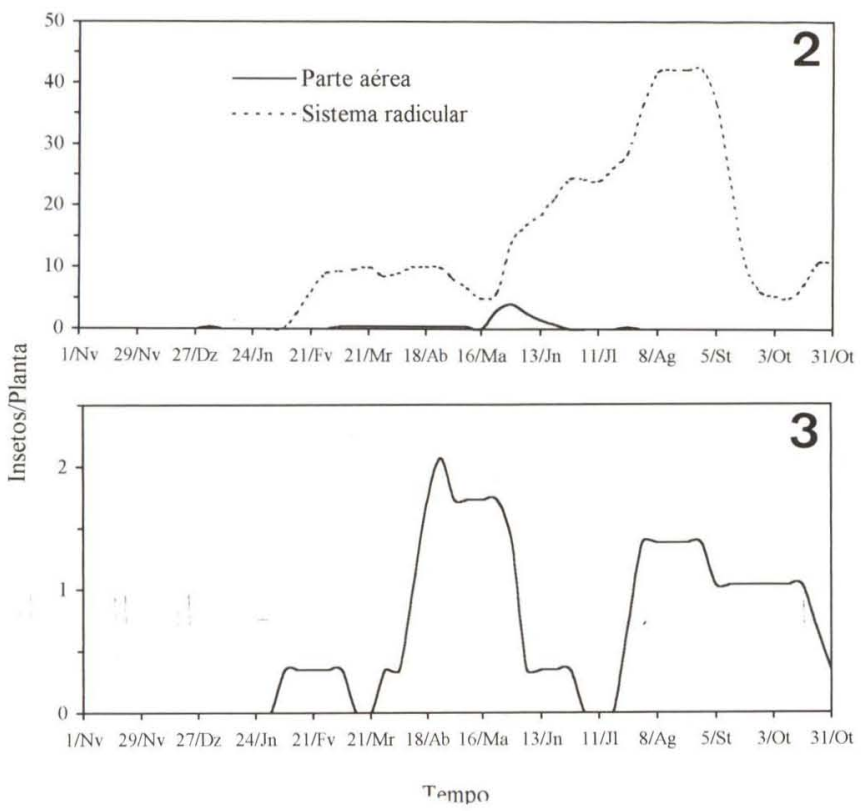

Figs 2-3. Flutuação populacional de himenópteros em jiloeiro, Viçosa, Minas Geraıs, 1984 e 1985. (2) Solenopsis saevissima; (3) Wasmannia auropunctata no sistema radicular.

Tabela II. Correlações de Pearson significativas a $\mathrm{P}<0,05$ entre as densidades populacionais de Solenopsis saevissima, Mycocepurus smithi, Hypoponera sp. e Crematogaster sp. com elementos climáticos. Viçosa, Minas Gerais, 1984 e 1985.

\begin{tabular}{llcc}
\hline \multicolumn{1}{c}{ Inseto } & Elementos climatológicos & Correlação & Significância pelo Teste "t" \\
\hline Crematogaster sp. (parte aérea) & Temperatura do ar & 0,3347 & 0,0051 \\
& Precipitação pluvial & 0,3223 & 0,0068 \\
Crematogaster sp. (sistema radicular) & Umidade relativa do ar & 0,2831 & 0,0156 \\
& Precipitação pluvial & $-0,2259$ & 0,0441 \\
Mycocepurus smithi & Temperatura do ar & $-0,3548$ & 0,0031 \\
& Precipitaçăo pluvial & $-0,4480$ & 0,0002 \\
Hypoponera sp. & Insolaçăo & 0,2929 & 0,0490 \\
& Temperatura do ar & $-0,7771$ & 0,0000 \\
Solenopsis saevissima (parte aérea) & Precipitaçăo pluvial & $-0,4104$ & 0,0007 \\
& Insolaçăo & 0,4303 & 0,0004 \\
Solenopsis saevissima (sistema radicular) & Temperatura do ar & Insolaçăo & 0,0161 \\
& Umperatura do ar & 0,2817 & 0,0377 \\
& Precipitação pluvial & $-0,7058$ & 0,0000 \\
& Insolaçăo & $-0,2611$ & 0,0239 \\
& & $-0,4481$ & 0,0002 \\
\end{tabular}

As maiores populações de Mycocepurus smithi Forel, 1893 (Fig. 3) e Wasmannia auropunctata (Roger, 1863) (Fig. 4) (Formicidae) foram verificadas no final dos cultivos. As densidades mais elevadas de M. smithi ocorreram em períodos de maior insolação, menores temperaturas e precipitações pluviais (Figs 1, 3, 4, Tab. II). Também DELLA LuCiA et al. (1982) verificaram que períodos de insolação mais elevada favorecem esta espécie. As densidades mais elevadas de Hypoponera sp. 


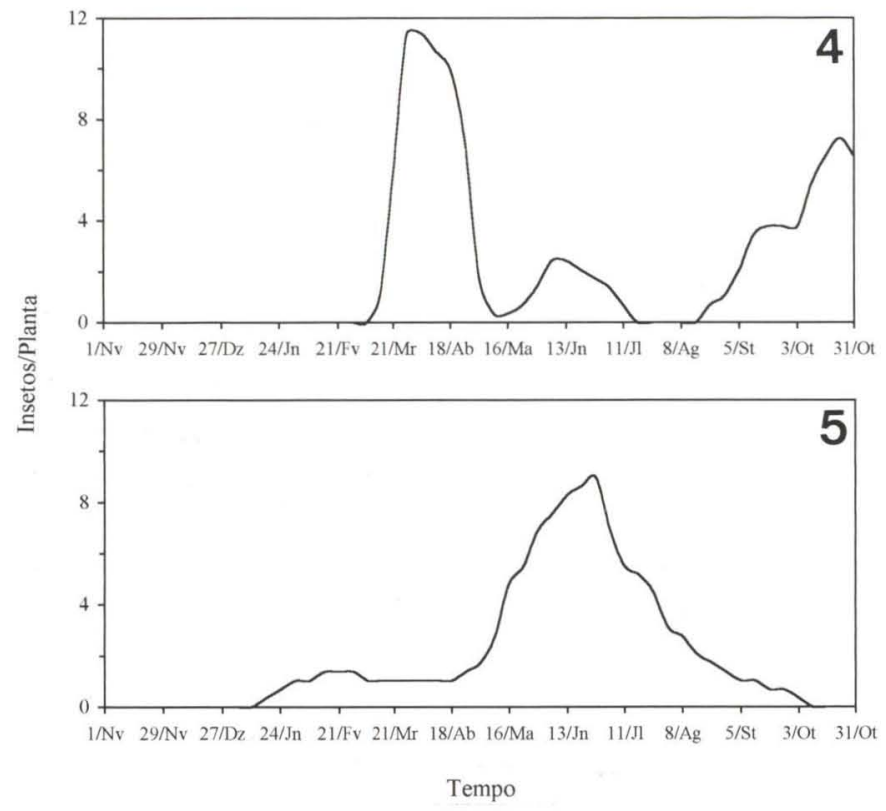

Figs 4-5. Flutuação populacıonal de himenópteros em jiioeiro, Viçosa, Minas Gerais, 1984 e 1985. (4) Wasmannia auropunctata no sistema radicular; (5) Hypoponera sp. no sistema radicular.

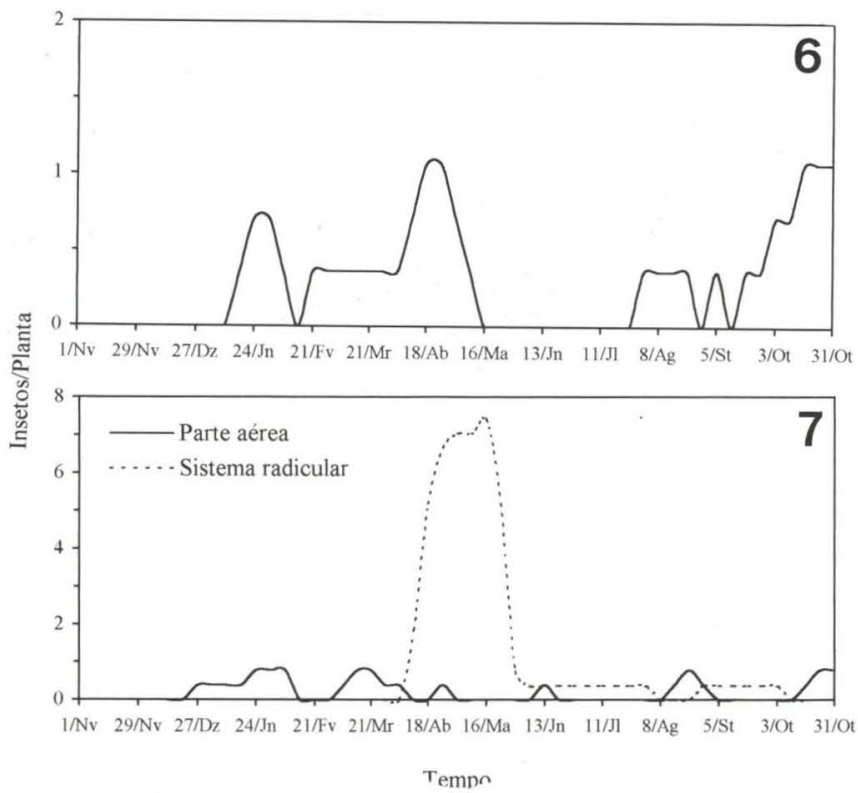

Figs 6-7. Flutuação populacional de himenopteros em jiloeiro, Viçosa, Minas Gerais, 1984 e 1985. (6) Camponotus rufipes na parte aérea de jiloeiro; (7) Crematogaster sp.. 
(Formicidae) ocorreram em período com ausência de chuvas, temperaturas baixas e elevada insolação (Figs 1, 5, Tab. II). Camponotus rufipes (Fabricius, 1775) (Formicidae) (Fig. 6) apresentou duas épocas de densidade populacional mais elevada, as quais ocorreram na fase final dos cultivos. Esta espécie foi observada em relação de protocooperação com Homoptera (Tab. I) das famílias Aethalionidae, Aphididae e Membracidae. Na relação de protocooperação, as formigas alimentamse dos restos fecais dos Homoptera promovendo sua higienização e protegendo-os da ação de predadores e parasitóides (FAllas \& Hilje 1985; SHutze \& MASCHWITZ 1991; CAMPBELl 1994; Willians \& FERRERO 1994). Crematogaster $\mathrm{sp.}$ (Formicidae) (Fig. 7) foi observada tanto na parte aérea das plantas como no sistema radicular. Na parte aérea foi observada relação de protocooperação com Homoptera, semelhante ao ocorrido com C. rufipes (Tab. I). No sistema radicular, sua densidade mais elevada, ocorreu ao final do primeiro cultivo, período de menor precipitação pluvial e maior umidade relativa do ar. (Figs 1, 7, Tab. II). Também RiBEIRO (1992) encontrou que a densidade de Formicidae em citros foi maior em períodos de maior Umidade relativa do ar. Na parte aérea das plantas a maior densidade desta espécie ocorreu em períodos de altas temperaturas e precipitações (Figs 1, 7, Tab. II).

Assim, verificou-se que as densidades mais elevadas da maioria das espécies de Formicidae ocorreram na fase final dos cultivos, em períodos de insolação elevada, baixas temperaturas e precipitações pluviais. A elevação das densidades populacionais destas espécies na fase final do cultivo, possivelmente esteja associada à maior disponibilidade alimentar, uma vez que é nesta época, que o sistema radicular das plantas, ao qual a maioria destas espécies estavam associadas, apresentava desenvolvimento mais elevado. A ação positiva da insolação sobre os Formicidae, possivelmente, se deve à ação reguladora deste elemento climático sobre reprodução, sobrevivência e desenvolvimento, sendo que tanto seu excesso como sua deficiência pode ser fator limitante para o inseto (SILVEIRA NeTO et al. 1976; PRICE 1984). O efeito negativo da elevação da temperatura do ar sobre os Formicidae pode estar relacionado ao fato de que altas temperaturas têm efeito negativo sobre a movimentação desenvolvimento e reprodução de insetos (SILVEIRA NeTO et al. 1976; ASHTON 1979, ANDERSEN 1983; PRICE 1984). Também DELla LuCia et al. (1982) (em milho, feijoeiro, milho-feijoeiro e pastagem); QUEIROZ (1991) (em cafeeiro) e RIBEIRO (1992) (em citros), verificaram que as densidades dos Formicidae foram maiores em temperaturas mais baixas. A elevação da densidade dos Formicidae em períodos de menor precipitação pluvial se deva, possivelmente, às chuvas terem efeito negativo sobre a dispersão (MAJER et al. 1982) e forrageamento (PORTER \& TSCHINKEL 1987) dos Formicidae.

\section{REFERÊNCIAS BIBLIOGRÁFICAS}

ANDERSEN, A.N. 1983. Species diversity and temporal distribution of ants in the semi-arid Malee region of Northwestern Victoria. Austr. Jour. Ecol. 8: 127237.

Ashton, D.H. 1979. Seed harvesting by ants in forests of Eucalyptus regans F. Muell in Central Victoria. Austr. Jour. Ecol. 4: 265-237. 
BuchmanN, S.L.; M.C. LoureIRo; L. ChANDLER; J.A.H. FreIRE; J.D. GALVÃo \& B. FERNANDES. 1983. Buzz pollination in angiosperms, p.73-113. In: C.E. JONES \& R.J. LITTLE. (Eds). Handbook of experimental pollination biology. New York, Van Nostrand Reinhorold Company, 588p.

CAMPBell, C.A.M. 1994. Homoptera associated with the ants Crematogaster clariventris, Pheidole megacephala and Tetramorium aculeatum (Hymenoptera: Formicidae) on cocoa in Ghana. Bull. Entomol. Res. 84 (3): 313-318.

Della LuCiA, T.M.C. 1982. Ordenação de comunidades de Formicidae em quatro agroecossistemas em Viçosa, Minas Gerais. Experientiae 28 (6): 67-94.

DENT, D. 1993. Insect pest management. Wallingford, Cab International, 604p.

FALLAS, F. \& L. HiLJE. 1975. Protocooperacion entre Aethalion reticulatum (L.) (Homoptera: Aethalionidae) y Camponotus abdominalis (F.) (Hymenoptera: Formicidae) en Costa Rica. Brenesia 24: 361-370.

FilgueirA, F.A.R. 1982. Manual de olericultura. São Paulo, Agronômica Ceres, $2^{\mathrm{a}}$ ed., $357 \mathrm{p}$.

FowleR, H.R. 1979. Notes on Labidus praedator (Fr. Smith) in Paraguay (Hymenoptera, Formicidae, Dorylinae, Ecitinini). Jour. Nat. Hist. 13 (1): 3-10.

Gallo, D.; O. NaKano; S. Silveira Neto; R.P.L. Carvalho; G.C. Batista; E. BERTI FILHO; J.R.P. PARRA; R.A. ZUCCHI; S.B. AlveS \& J.D. VENDRAMIM. 1988. Manual de Entomologia agrícola. São Paulo, Agronômica Ceres, 649p.

MaJer, J.D.; M. SARTori; R. STONE \& W.S. Perriman. 1982. Recolonization by ants and other invertebrates in rehabilitated mineral sand mines near Eneabba, Western Australia. Reclam. Reveget. Res. 1 (1): 63-81.

MENEZES, M. DE. 1978. Notas do hábito de oviposição e plantas hospedeiras de Apagonia grossa (Signoret, 1854) (Homoptera: Cicadellidae: Cicadellinae). Revta bras. Ent. 22 (2): 61-64.

MiCHERNER, C.D. 1962. An interesting method of pollen collecting by bees from flowers with tubular anthers. Rev. Biol. Trop. 10 (2): 167-175.

PEDIGO, L.P. 1989. Entomology and pest management. New York, Macmillan Publishing Company, 646p.

PorTER, S.D. \& W.R. Tschinkel, W.R. 1987. Foraging in Solenopsis invicta (Hymenoptera, Formicidae): effects of weather and season. Env. Entomol. 16: 802-808.

PRICE, J.W. 1984. Insect ecology. New York, John Wiley \& Sons, 2 ed. 406p.

QueIroz, M.V.B. 1991. Comunidades de gêneros edáficos de Formicidae (Insecta, Hymenoptera), em ecossistemas de Coffea arabica. Tese de Mestrado, não publicada, Universidade Federal de Viçosa, Viçosa, 87p.

RIBEIRO, J.D. 1992. Efeito de práticas culturais sobre formicídeos edáficos (Hymenoptera: Formicidae) em ecossistemas de citros. Tese de Mestrado, não publicada, Universidade Federal de Viçosa, Viçosa, 98p.

SAntos, G.P. \& A.C.Q. PInTo. 1981. Biologia de Cycloneda sanguinea e sua associação com pulgão em mudas de mangueira. Pesq. Agropec. Bras. 16 (4): 473-476.

SChUtZE, M.; MASChwitZ, U. 1991. Enemy recognition and defense within tro- 
phobiotic associations with ants by the soldier caste of Pseudoregma sundanica (Homoptera: Aphididae). Entomol. Gen. 16 (1): 1-12.

Silveira, F.A. \& M.J.O. Campos. 1995. A melissofauna de Corumbataí (SP) e Paraopeba (MG) e uma análise da zoogeografia das abelhas do cerrado brasileiro (Hymenoptera, Apoidea). Revta bras. Ent. 39 (2): 371-401.

Silveira Neto, S.; O. Nakano; D. Barbin \& N.A. Villa Nova. 1976. Manual de ecologia dos insetos. Piracicaba, Agronômica Ceres, 419p.

Villas-BôAs, G.L. \& M. CAstelo Branco. 1990. Manejo Integrado de Pragas em Hortaliças. Anais do Simpósio de Manejo Integrado de Pragas e Nematóides, Jaboticabal, 1: 147-150.

Williams, D.J. \& D. Matile Ferrero. 1994. A new genus and species of mealybug (Homoptera: Coccoidea: Pseudococcidae) associated with ants in swollen thorn acacias in Tanzania. Ann. Soc. Entomol. France 30 (3): 273-277.

Recebido em 16.I.1997; aceito em 18.IX.1997. 